\title{
Antiochus Epiphanes und der epiphane Gott. Gefühle, Emotionen und Affekte im Zweiten Makkabäerbuch
}

\author{
BARBARA SCHMITZ
}

Das Zweite Makkabäerbuch ist eine absichtsvoll komponierte Erzählung, die ihr Erzählinteresse in der Vorrede (2Makk 2,19-32) und im Epilog (2Makk 15,37-39) benennt. Die Erzählung soll - so die Selbstauskunft der Erzählung im Vorwort - den Leserinnen und Lesern zur

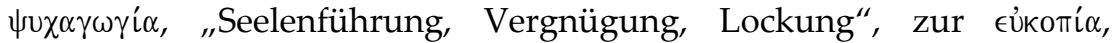
„Leichtigkeit“ sowie zur $\omega \dot{\phi} \hat{\epsilon} \lambda \epsilon \iota \alpha$, „Vorteil, Nutzen, nützlichen Kenntnis" dienen (2Makk 2,25). ${ }^{1}$ Damit präsentiert sich das Zweite Makkabäerbuch als eine absichtsvolle literarische Erzählung, die ganz bewusst bei den Leserinnen und Lesern emotionale Wirkung, Gefühle und Affekte hervorrufen will. ${ }^{2}$

Dieses Ziel wird gleich zu Beginn der Erzählung auf der Kommunikationsebene zwischen Erzählstimme ${ }^{3}$ und Lesenden benannt. Das auf diese Weise offengelegte Vorhaben der Erzählung lässt zunächst fragen, wie die emotionale Wirkung bei den Leserinnen und Lesern erzielt werden soll. Darüber hinaus ist die viel grundlegendere Frage zu stellen, warum es für das Zweite Makkabäerbuch von so zentralem Interesse ist, Emotionen bei den Rezipienten hervorzurufen.

1 Vgl. zu den Intentionen hellenistischer Geschichtsschreibung die Belege bei DORAN, Temple Propaganda, 79.

2 So auch NICKLAS, Historiker, 80.

3 Zum Terminus „Erzählstimme“ und der Analyse der Erzählstimme vgl. SCHMITZ, Prophetie, 21-42. Zum Vorwort vgl. auch HERR, Standpunkt, 1-31. 


\section{Der seleukidische König Antiochus im Zweiten Makkabäerbuch}

Die Figur von Antiochus Epiphanes tritt zwischen 2Makk 4,7 und 10,9 in verschiedenen Erzählsequenzen auf, die ihn - wie die einzelnen Steine eines Mosaiks - porträtieren. ${ }^{4}$

Antiochus tritt zu Beginn als Verhandlungspartner von Jason auf, dem Bruder des Hohepriesters Onias, dem es gelingt, das Amt des Hohepriesters von Antiochus zu erkaufen (2Makk 4,7-9). Jason leitet umfangreiche Maßnahmen in Jerusalem ein (2Makk 4,10-12), die zu einer

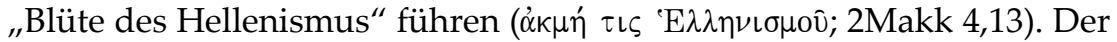
zeitgleiche Amtsantritt der beiden Figuren scheint kein Zufall zu sein, vielmehr passen der neue König und der neue Hohepriester insofern

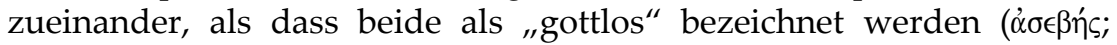
2Makk 4,13; 10,10). Damit hebt sich die mit Antiochus und Jason beginnende neue Phase in der Geschichte Judäas von der vorangehenden

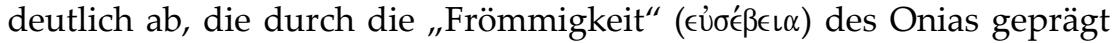
war (2Makk 3,1). Durch den synchronisierten Herrschaftsbeginn von Antiochus als König des Seleukidenreichs und Jason als Hohepriester in Jerusalem wird ein politischer Einschnitt markiert.

Bei den weiteren Ereignissen wird der seleukidische König als geldorientiert und geldgierig porträtiert. So überträgt Antiochus das Jerusalemer Hohepriesteramt an den Meistbietenden: zuerst an Jason (2Makk 4,8-9), nach drei Jahren an Menelaus, der Antiochus eigentlich die von Jason versprochenen Gelder überbringen soll, dem es aber gelingt, das Hohepriesteramt durch höhere finanzielle Versprechungen aus den Geldreserven des Tempels selbst zu erlangen (2Makk 4,23-25). Antiochus seinerseits raubt aus dem Jerusalemer Tempel wertvolle Geräte und 1800 Talente (2Makk 5,16.21).

Neben seiner Geldgier und Bestechlichkeit sind Brutalität und Grausamkeit weitere hervorstechende Charakteristika von Antiochus,

4 Man kann das Zweite Makkabäerbuch nach den jeweils regierenden seleukidischen Königen gliedern: Während der erste Teil der Handlung noch unter Seleukus (IV.) stattfindet (2Makk 3,1-40; 4,1-6), spielt der zweite, längere Abschnitt der Erzählung (2Makk 4,7-10,9) unter der Regentschaft des Antiochus Epiphanes. Dieser wird in der Erzählung nur im Vorwort (2Makk 2,20) und zu Beginn und am Ende seiner Regierungszeit mit seinem Beinamen als „Antiochus Epiphanes“ bezeichnet (2Makk $4,7 ; 10,9.13)$. Der dritte Abschnitt findet unter den Königen Antiochus Eupator (2Makk 10,10-13,26) und Demetrios (2Makk 14,1-15,36) statt, die aber - wie Seleukos im ersten Abschnitt - für die Handlung nicht die zentrale Bedeutung haben wie Antiochus Epiphanes im mittleren Abschnitt. Aufgrund der besonderen Bedeutung der Figur des Antiochus ist die Analyse seiner Präsentation besonders wichtig. 
von denen im Zweiten Makkabäerbuch in unterschiedlichen Konstellationen - sowohl in kriegerischen Auseinandersetzungen als auch in einzelnen Folterszenen - ausführlich berichtet wird. Im Kontext seines Feldzugs gegen Ägypten wird erzählt (2Makk 5,1), dass er Jerusalem

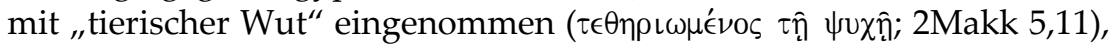
gnadenlos $(\dot{\alpha} \phi \in\llcorner\delta \hat{\omega} \varsigma)$ ein großes Blutbad angerichtet hat und ohne Unterschiede alt und jung, insgesamt $80.000^{5}$ Menschen, hat abschlachten, ermorden oder in die Sklaverei verkaufen lassen (vgl. 2Makk 5,12-14). Der Höhepunkt des Wütens in Jerusalem wird erreicht, als Antiochus unter der Führung von Menelaus „den heiligsten Tempel der ganzen

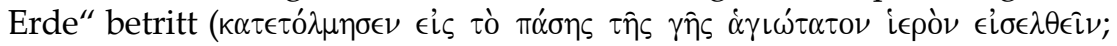
2Makk 5,15). Mit seinen „unreinen Händen“ macht er sich an den heili-

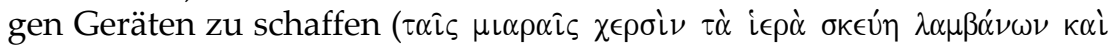

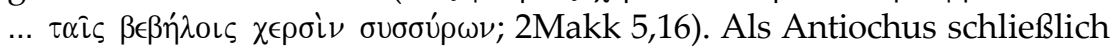
auch noch die Schatzkammer des Tempels betritt, geschieht auffälligerweise nichts. Dies ist höchst erstaunlich, denn dies ist im Zweiten Makkabäerbuch das dritte Mal, dass vom unerlaubten Betreten der Schatzkammer des Jerusalemer Tempels berichtet wird, aber beim ersten Mal Heliodor durch eine Epiphanie Gottes (vgl. 2Makk 3,24-26) und beim zweiten Mal Lysimachus durch Jerusalemer vertrieben wurden (vgl. 2Makk 4,43). Es bedarf deshalb einer Erklärung, weshalb diesmal nichts geschieht. Daher wird die Erzählung unterbrochen, um in einem ausführlichen Kommentar zu reflektieren, warum Gott bewusst darauf verzichtet habe, Antiochus aufzuhalten (vgl. 2Makk 5,17-20). Die zentrale Grundargumentation hierbei ist, dass Gott das Volk nicht wegen der Stätte, sondern die Stätte um des Volkes willen ausgesucht habe (2Makk 5,19). Signifikant ist, dass in einer Erzählsequenz, die von dem Nichteingreifen Gottes in der Not und dem Erfolg von Antiochus berichtet, Antiochus als „überheblich" charakterisiert

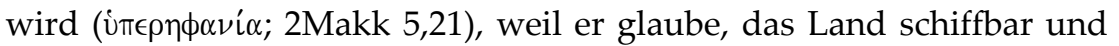
das Meer begehbar machen zu können (vgl. auch 2Makk 9,8.10).

Nach der Schilderung des Angriffs auf Jerusalem im Kontext des zweiten Ägyptenfeldzugs des Antiochus werden in 2Makk 6 und 7 eindrücklich und ausführlich die kultischen Neuerungen von Antiochus geschildert, die die Martyrien der toratreuen Jüdinnen und Juden zur Folge haben. Nach dem Zweiten Makkabäerbuch ist es Antiochus, der befiehlt, dass sich die jüdische Gemeinschaft von ihrem väterlichen Glauben abwenden und nicht mehr nach den Geboten Gottes leben sol-

5 Diese Angabe dürfte die Zahl der damaligen Gesamtbevölkerung von Jerusalem bei weitem übertreffen, so SCHWARTZ, 2 Maccabees, 259. 
le. ${ }^{6}$ Auf seine Initiative sei der Jerusalemer Tempel in einen Tempel für "Zeus Olympios" umgeweiht worden, und es sei Juden verboten gewesen, den Sabbat zu feiern und die Feste zu begehen, ja überhaupt, sich als Jude zu bekennen (2Makk 6,6). Zu all dem wurden sie auch noch gezwungen, an heidnischen Opfern teilzunehmen und fremden Göttern zu opfern (2Makk 6; 7). Diese Forderungen, die im Zweiten Makkabäerbuch Antiochus zugeschrieben werden, rufen unter den Jüdinnen und Juden großen Protest sowie die sich um Judas Makkabäus formierende Guerillatruppe hervor.

Zwischen der ersten Erwähnung von Judas in 2Makk 5,27 und dem Beginn des militärischen Widerstands in 2Makk 8 sind just die Martyrienschilderungen eingefügt, ${ }^{7}$ die zum einen den Mut und die Standhaftigkeit der Jüdinnen und Juden schildern und die zum anderen aber die Grausamkeit des Antiochus beleuchten. Dabei folgt auf die ersten beiden kurzen Martyriumsschilderungen, die sich jeweils an den Kennzeichen jüdischer Identität entzünden (Beschneidung und Sabbat), ${ }^{8}$ ein ausführlicher Erzählkommentar (2Makk 6,12-17). Erst danach fährt die Erzählung mit den beiden langen Martyrien von Eleasar (2Makk 6,18-31) und den sieben Brüdern und ihrer Mutter (2Makk 7) fort. Bevor also die großen Martyrien erzählt werden, werden diese den explizit angesprochenen Lesenden gedeutet. Dabei wird das Leiden des Volkes in den Horizont der Pädagogik Gottes, seines Erbarmen für das Volk und seiner Gerechtigkeit mit Blick auf die Täter gestellt. Die Täter

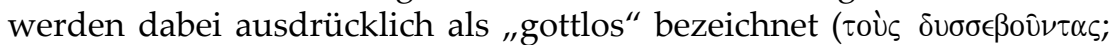
2Makk 6,13). Während Antiochus beim Martyrium Eleasars selbst nicht zugegen ist (2Makk 6,18-31), ist er während des Martyriums der sieben Brüder und ihrer Mutter nicht nur anwesend, sondern sogar der Initiator der Folterungen: Er ist es, der die Brüder zwingen will, Schweinefleisch zu essen, der sie geißeln und auspeitschen, ihnen die Zunge abschneiden, die Kopfhaut abziehen und sie verstümmeln lässt (2Makk 7,1.4). So lässt er einen Bruder nach dem anderen vor den Augen der übrigen Brüder und ihrer Mutter ermorden. Die Unerschrockenheit der Brüder bleibt jedoch nicht ohne Wirkung. So ist selbst der König über

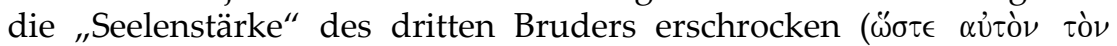

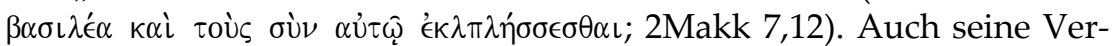
suche, den siebten Bruder durch großzügige Versprechungen zum Ab-

6 Vgl. hierzu WeITZMAN, Antiochus's Persecution, 219-34.

7 Zu den Martyrien vgl. v.a. VAN HENTEN, Martyrs; VAN HENTEN, Selbstverständnis, 127-61.

8 Beispielartig werden zwei Frauen, die ihre Söhne beschneiden lassen (2Makk 6,10), und solche, die heimlich den Sabbat feiern (2Makk 6,11), genannt. 
fall von Gott zu bewegen $(2$ Makk 7,24$)$ und die Mutter zu beeinflussen, ihren siebten und letzten Sohn zu retten (2Makk 7,25), bewirken genau das Gegenteil: Wie bereits die anderen Söhne zuvor, bestärkt die Mutter auch ihren letzten Sohn, in den Tod zu gehen (vgl. 2Makk 7,26-29). Die Söhne jedoch charakterisieren unter Folter den König: So spricht der zweite Sohn den König als "Verbrecher" an ( $\dot{\alpha} \lambda \dot{\alpha} \sigma \tau \omega \rho$; 2Makk 7,9) und der fünfte nennt ihn einen „vergänglichen Menschen“

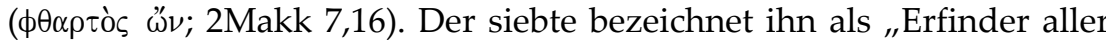

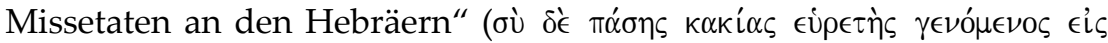

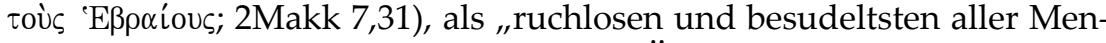
schen“9 (2Makk 7,34), der die seiner „Überheblichkeit“ gebührende

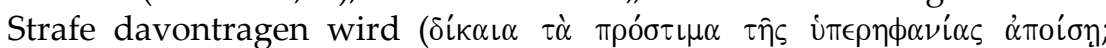
2Makk 7,36 ) und stellt ihn dem lebendigen, allmächtigen und einzigen Gott gegenüber (2Makk 7,31-38). ${ }^{10}$ So bleibt der König, der außer sich

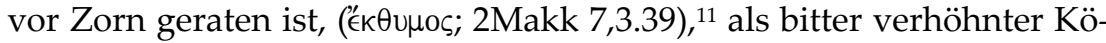

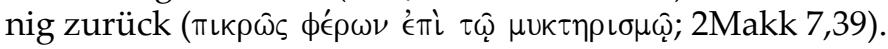

Die im Erzählkommentar in den Martyrien angekündigte Strafe für Antiochus (2Makk 6,12-17) und sein vom vierten, fünften und sechsten Bruder angekündigter Tod (2Makk 7,14.17.19) werden in 2Makk 9 erzählt. ${ }^{12}$ Dazwischen ist die Erzählung über den Beginn des Widerstands unter Judas eingefügt (2Makk 8), der den in 2Makk 5,27 eröffneten Erzählfaden aufnimmt.

Die Schilderung des Todes von Antiochus entspricht freilich nicht den historischen Gegebenheiten, sondern ist eine literarische Inszenierung für die Aussageabsicht des Zweiten Makkabäerbuches. Wie bereits zuvor in Jerusalem (2Makk 5,15-16) versucht Antiochus in Persepolis, sich eines Teils des Tempelvermögens zu bemächtigen; anders als in Jerusalem scheitert er jedoch am Widerstand der Bevölkerung (2Makk 9,2). Bei seinem Rückzug hört er in Ekbatana zudem von den in 2Makk 8,30-33 geschilderten Ereignissen in Judäa. In seinem Ärger über den Widerstand in Persepolis reagiert er, wie bereits in 2Makk

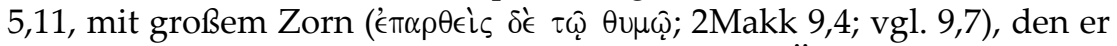
an den Juden auslassen will. Wieder wird seine "Überheblichkeit" ge-

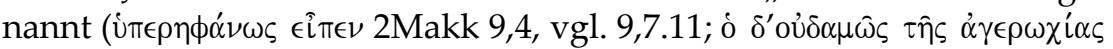
" $\lambda \eta \gamma \in \nu ; 2$ Makk 9,7), aus der heraus er ankündigt, Jerusalem in ein „Mas-

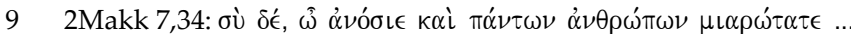

10 Da jedoch betont wird, dass die Brüder in der Sprache ihrer Väter sprechen (vgl. 2Makk 7,8 vgl. 7,21.27), ist von der erzählerischen Konstruktion der Szene unklar, ob der König sie verstehen kann.

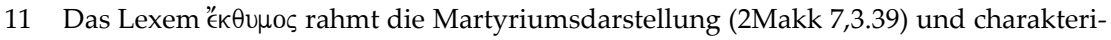
siert Antiochus als "̌́ $\theta$ vuo

12 Zu 2Makk 9 vgl. v.a. NICKLAS, Historiker, 80-92; SCHWARTZ, Antiochus, 257-65. 


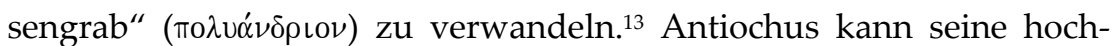
fliegenden Pläne aber nicht mehr realisieren, denn: „Der alles überschauende Herr, der Gott Israels, traf ihn mit einem unheilbaren und

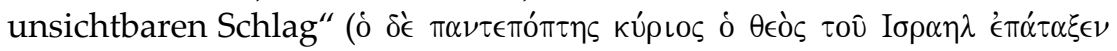

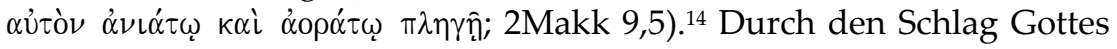
trifft nun Antiochus all das, was er Anderen angetan hat. ${ }^{15}$ Dabei werden zahlreiche Lexeme und Motive aus den in 2Makk 6 und 7 geschilderten Martyrien aufgenommen, vor allem die ausführlichen Ankündigungen eines qualvollen Todes des Antiochus durch den siebten und letzten Sohn. ${ }^{16}$

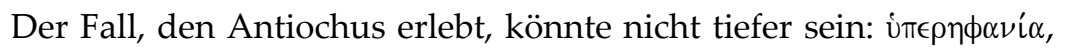
„Überheblichkeit" wird in der Darstellung von Antiochus zum Grundmotiv. In 2Makk 5 war davon die Rede, dass Antiochus in seiner i $\pi \in \rho \eta \phi \alpha \nu i \alpha$, „Überheblichkeit" glaube, das Land schiffbar und das Meer begehbar machen zu können (2Makk 5,21); nun heißt es, dass Antiochus glaube, den Wogen des Meeres gebieten, die Höhe des Gebirges mit einer Waage abwägen (2Makk 9,8) und nach den Sternen greifen zu können (2Makk 9,10). Durch diese Aussagen und deren intertextuellen Bezüge zu Jes 40,12; Ijob 38 oder Ps 65,8; 90,10 wird Antiochus als jemand gezeichnet, der beansprucht gottgleich zu sein. ${ }^{17}$ Dieses Bestreben wird Antiochus - wenn auch in (vermeintlich) reuiger Umkehr selbst in den Mund gelegt (vgl. 2Makk 9,12).

Nicht ohne ironischen Unterton kostet die Erzählung das Menschsein des Antiochus in der Realität seines Sterbens aus: Der Gestank, der von dem von Würmern zerfressenen Antiochus ausgehe, sei unerträglich und verpeste das ganze Lager (2Makk 9,10). Der vor Verwesung bereits lebend stinkende Antiochus erweist sich damit als Mensch und eben nicht als Gott!

Anders jedoch - so die Erzählung - ist das Selbstbild des Antiochus, der sich als „wohltätig“" gegenüber Juden ( $\epsilon$ ưvo $\alpha$; 2Makk 9,26), als

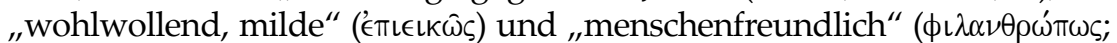
2Makk 9,27) beschreibt. Damit sind entscheidende Charakteristika ei-

13 Vgl. zu 2Makk 9,7 auch SCHWARTZ, Antiochus, 257-65.

14 Das Motiv, dass der Schlag Gottes nicht zu sehen sei, entspricht einem Motiv, das sich durchgehend in den Epiphanien findet, nämlich dass diese immer nur von bestimmten, nie aber von allen Beteiligten gesehen werden können; vgl. die Tabelle in SCHMITZ, Auferstehung, 105-42.

15 Vgl. hierzu EGO, God's Justice, 141-54.

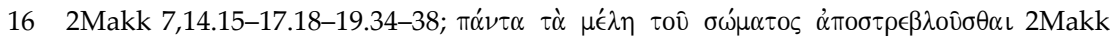

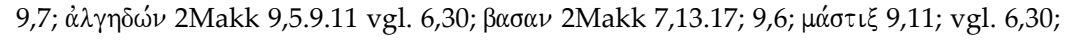
7,1.37 etc.

17 Vgl. hierzu v.a. NiCKLAS, Historiker, 85. 
nes idealen Königs in hellenistischer Zeit genannt, die Antiochus für sich beansprucht, die in der Erzählung aber in einem Kontrast zum Verhalten des Antiochus stehen.

An nur zwei Stellen findet sich im Zweiten Makkabäerbuch ein abweichendes Bild, bei dem positivere Züge Antiochus' erzählt werden. Die erste findet sich in 2Makk 4, als Antiochus von Mitleid und Trauer wegen der Ermordung des Onias ergriffen ist und um ihn

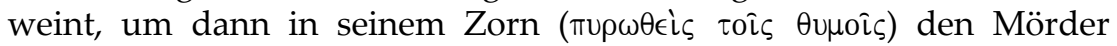
Andronikus zu degradieren und umzubringen (2Makk 4,37-38). Antiochus' Trauer überrascht, weil er selbst Onias nicht unterstützt, sondern durch Jason ersetzt hatte. Jedoch scheint diese Szene nicht in erster Linie dazu zu dienen, Antiochus positiv zu schildern, sondern vielmehr seine irrationale Emotionalität zu verdeutlichen, die sich als unkontrollierbar und gefährlich erweist. Es ist nicht der gerechte Zorn des guten Königs, dessen Aufgabe es ist, Unrecht zu verfolgen und zu ahnden, sondern es wird vielmehr als die eruptive Reaktion eines Herrschers geschildert, dessen Zorn gefährlich ist - wie es sich in der Erzählung auch beim Martyrium der sieben Brüder und ihrer Mutter zeigen wird (2Makk 7,3.39).

Die zweite Stelle, an der Antiochus auf den ersten Blick positiv geschildert wird, findet sich in 2Makk 9. Angesichts seiner Qualen und seines nahenden Todes lässt Antiochus von seiner "Überheblichkeit"

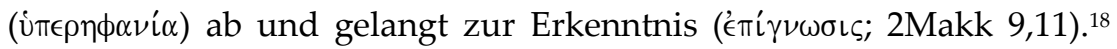
Worin diese Erkenntnis besteht, wird in 2Makk 9,12 in einer Rede Antiochus selbst in den Mund gelegt: „Es ist Recht, sich Gott zu unterwerfen und als Sterblicher sich nicht gottgleich (iøó $\theta \in \alpha$ ) zu dünken" (2Makk

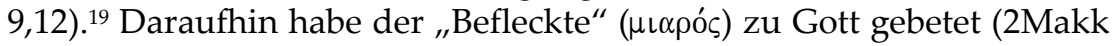
9,13-17). Das indirekt wiedergegebene Gebet endet mit dem Wunsch, selbst Jude werden zu wollen (2Makk 9,17). Nicht nur die Darstellungsweise, die auf Distanz zu Antiochus geht, sondern auch die szenische Einbettung sprechen dafür, diesen Wunsch als der aktuellen Not geschuldet und als vorgetäuscht zu verstehen. ${ }^{20}$ Schließlich wird Antiochus nicht - wie z.B. Heliodor (vgl. 2Makk 3), der auch wie Antiochus die Schatzkammer des Tempels betreten hat - von Gott gerettet. Gott greift gerade nicht zur Rettung von Antiochus ein, stattdessen wird mehrfach betont, dass Antiochus mit seinem Ende die gerechte Strafe

18 In der szenischen Konstellation wird - mit umgekehrtem Vorzeichen - das Martyrium der Brüder aktualisiert: Wie diese angesichts ihrer von Antiochus zugefügten Folter jeweils den Gott Israels bekennen, so bekennt nun Antiochus unter der ihm vom Gott Israels zugefügten Qual diesen als den einzigen und wahren Gott.

19 Vgl. hierzu auch HABICHT, 2. Makkabäerbuch, 245.

20 So NICKLAS, Historiker, 90-91; anders SCHWARTZ, 2 Maccabees, 338. 
für das erhalten habe, was er anderen angetan hatte (2Makk 9,4.6.18. 28).

Blickt man auf den Verlauf der Erzählung und die Art und Weise, wie in ihr Antiochus geschildert wird, dann fällt auf, dass sich anfangs bei der Einführung der Figur zunächst keine oder kaum wertende oder emotionale Äußerungen finden, diese aber in der Erzählung zunehmen und sich in 2Makk 7 und 9 konzentrieren: Während Antiochus in 2Makk 7 von den sieben Brüdern und der Mutter charakterisiert wird, wird Antiochus in 2Makk 9 von der Erzählstimme als „überheblich“

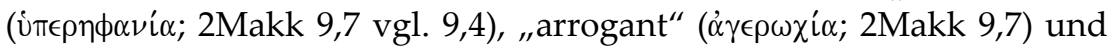
„prahlerisch" ( $\dot{\alpha} \lambda \alpha \zeta$ oveí ; 2Makk 9,8) beschrieben; zudem wird er be-

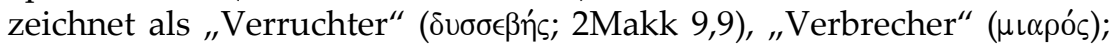

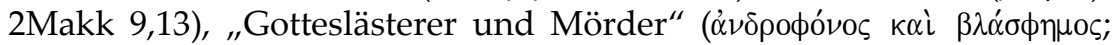
2Makk 9,28).

Somit kann man in der Erzählung eine sich steigernde Linie und eine zunehmende Emotionalität in der wertenden Beschreibung des Antiochus aufzeigen. Während er zu Beginn in erster Linie implizit durch seine Handlungen charakterisiert wird, finden sich zunehmend direkte Wertungen.

Damit ist jedoch die Frage nach dem Warum offen: Was veranlasst das Zweite Makkabäerbuch zu dieser hohen und intensiven Emotionalität in der Schilderung des seleukidischen Königs?

In der Darstellung des Antiochus im Zweiten Makkabäerbuch kann man eine aufschlussreiche Beobachtung machen, die nur auf den ersten Blick ein Detail zu sein scheint: Der König wird in der gesamten Erzählung als "der König“ oder mit seinem Eigennamen "Antiochus" bezeichnet. Aber nur an ganz wenigen, herausgehobenen Stellen wird er

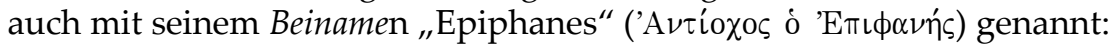
bei seiner Einführung im Vorwort in 2Makk 2,20, bei seinem ersten Auftritt in der Erzählung in 2Makk 4,7 und bei seinem Tod in 2Makk 10,9.13. Es zeigt sich somit, dass die Verwendung des Epithetons „Epiphanes“ nur an wenigen, sorgsam ausgewählten Stellen erfolgt. Wie kommt der König Antiochus zu diesem theologischen Beinamen? Interessanterweise ist mit dem Beinamen Epiphanes auf historischer Ebene ein Anspruch verbunden, den Antiochus für sich reklamiert und öffentlichkeitswirksam inszeniert hat. Exemplarisch lässt sich dies auf den neuen, unter Antiochus geprägten Münzen aufzeigen. Daher wird im Folgenden die historische Person von Antiochus und seine Inszenierung als „Epiphanes“ am Beispiel der von ihm durchgeführten Reform des Münzwesens vorgestellt. 


\section{König Antiochus Epiphanes und die Reform des seleukidischen Münzwesens}

Antiochus IV. (175-164 v.Chr.) ist der jüngste Sohn von Antiochus III. und dürfte zwischen 218 und 200 v.Chr. geboren sein. ${ }^{21}$ In antiken Quellen wird er erstmals im Friedensvertrag von Apameia (189 v.Chr.) erwähnt, in dem die Römer von den Seleukiden neben ernormen Reparationszahlungen auch zwanzig Geiseln fordern. Zu diesen Geiseln gehört Antiochus, der seine Jugend als königliche Geisel in Rom verbringt. Um 178 v.Chr. wird Antiochus gegen Demetrios, den Sohn seines amtierenden Bruders Seleukus IV., ausgetauscht. Daraufhin begibt er sich nach Athen. Als 175 v.Chr. Seleukus IV. von seinem General Heliodor ermordet und dieser für den noch minderjährigen Sohn des Seleukus, Antiochus ( $\Sigma \in \lambda \in$ $\epsilon$ коu), die Herrschaft übernimmt, gelingt es Antiochus mit Unterstützung von Eumenes II., dem König von Pergamon, in weniger als zwei Monaten die politische Lage im Seleukidenreich für sich zu entscheiden und den seleukidischen Thron einzunehmen. Er heiratet seine Schwägerin Laodike, erkennt ihren Sohn Antiochus Seleukou als seinen Sohn an und übernimmt nun seinerseits - zunächst in Stellvertretung für den noch Minderjährigen - die Herrschaft im Seleukidenreich. ${ }^{22}$

Nach der Machtübernahme ist es Antiochus' erstes Ziel, seine Herrschaft zu stabilisieren und erste Erfolge vorzuweisen. Auf diese Erfolge ist er dringend angewiesen, um seine Herrschaft $\mathrm{zu}$ legitimieren. ${ }^{23}$ In der Außenpolitik baut Antiochus seine diplomatischen Beziehungen gerade gegenüber Rom und Athen aus und zeigt sich als "Wohltäter"

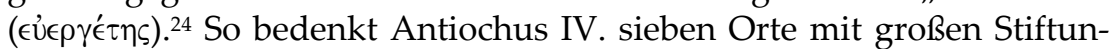

21 Vgl. zum folgenden MitTAG, Antiochos.

22 BUNGE, Theos, 60, weist daraufhin, dass die Übernahme der Vormundschaft für den minderjährigen Neffen den traditionellen Interessen der seleukidischen Dynastie entsprach. Vgl. auch FUNCK, Herrscherkult, 402-07; MITTAG, Antiochos 128-39.

23 Hellenistische Herrschaft legitimiert sich im Wesentlichen durch den Erfolg, den der König vorzuweisen hat, vgl. GEHRKE, König, 247-77.

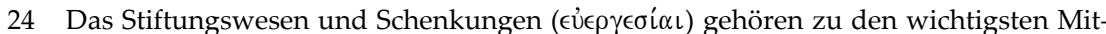
teln der propagandistischen Selbstinszenierung, sollen Resonanz und Aufmerksamkeit in der Weltöffentlichkeit erzeugen und haben die Funktion, einen "guten" König von einem Tyrannen zu unterscheiden. Als Wohltäter ( $\left.\left.\epsilon \cup \in \in \rho \gamma^{\prime} \in \tau\right\rceil \varsigma\right)$ stiftet der König den Städten Schenkungen, Steuersenkungen oder sonstigen Begünstigungen, was das Prestige ( $\left.\delta \xi^{\prime} \alpha\right)$ und die Anerkennung des Königs fördert. Darin zeigt er sein Wohlwollen $(\epsilon \cup ้ \nu \circ\llcorner\alpha)$ und erweist seine $\phi\llcorner\lambda \alpha \nu \theta \rho \omega \pi i \alpha$, worauf die Beschenkten mit Loyalität $(\epsilon \cup ̋ \nu \circ\llcorner\alpha)$ und Wertschätzung ( $\tau \iota \mu \eta ́)$ reagieren. Dieses reziproke Verhältnis ist eine wesentliche Grundlage hellenistischer Herrschaft. Zugleich müssen Misserfolge kaschiert werden: So veranstaltete Antiochus IV. 166 v.Chr. das einen Monat 
gen. ${ }^{25}$ Zugleich muss er seine Macht nach innen gegenüber den unterschiedlichen Gruppen im seleukidischen Reich sichern. So ist anzunehmen, dass ihm der Tod seines Neffens und eigentlichen Königs nicht ungelegen kommt, der im Jahr 170 unter ungeklärten Umständen stirbt. ${ }^{26}$ Es war, besonders solange die Söhne von Seleukos IV. noch am Leben waren, ein zentrales Anliegen Antiochus' IV., seine Herrschaft $\mathrm{zu}$ legitimieren und $\mathrm{zu}$ sichern. Ein wichtiges Mittel hierzu waren die von ihm neu emittierten Münzen.

Die Reform des Münzwesens ${ }^{27}$ erfolgt nach der Begleichung der letzten im Friedensvertrag von Apameia festgesetzten Rate der Reparationszahlungen an Rom. Diese Münzreform ist besonders gut in Antiochia am Orontes, dem wichtigsten Herrschafts- und Wirtschaftszentrum im seleukidischen Reich, aufzuzeigen, weil sich bei der Münzstätte in Antiochia eine deutliche staatliche Lenkung nachweisen lässt, die in anderen Münzstätten im Reich nicht im selben Maß gegeben ist. Die Reform betrifft zunächst die Tetradrachmen, deren Gewicht Antiochus auf 17,0, später auf 16,7 Gramm reduzieren ließ.28 Weitere Wirtschaftsreformen leitet Antiochus auf dem Höhepunkt seiner Macht nach dem ersten Feldzug des sechsten Syrischen Krieges (169/168) ein, zu denen auch eine Reform der Bronzemünzen gehörte. Beide Münztypen lässt Antiochus zudem mit neuen Motiven und Aufschriften versehen und ist mit den Tetradrachmen auf internationaler Ebene, mit den Bronzemünzen stärker im Alltag der Menschen präsent. Von daher lässt sich zu Recht von den Münzen als einem Massenmedium der Antike sprechen. ${ }^{29}$ Durch die weite Verbreitung der Münzen in unterschiedlichen Kreisen der Bevölkerung und Kontexten hat die Münzprägung eine nicht zu unterschätzende symbolische Bedeutung, mit

dauernde, größte Spektakel der hellenistischen Geschichte in Daphne bei Antiochia (166 v.Chr.), das nicht zufällig nach dem „Tage von Eleusis“, dem von Rom erzwungenen Rückzugs aus Ägypten im Jahr 168 v.Chr., stattfand.

25 In Athen ließ er z.B. den Zeus-Olympios-Tempel neu erbauen, übernahm den größten Teil der Kosten für die Erneuerung der Stadtmauern in Megalopolis, zahlte in Tegea ein Theater und auf Delos einen Altar und Statuen etc. Mit diesen Stiftungen übertrifft Antiochus die zeitgleich regierenden Könige der hellenistischen Welt um ein Vielfaches.

26 Antiochus hat für den Tod seines Neffen den Andronikus verantwortlich gemacht, der nach dem Zweiten Makkabäerbuch wegen der Ermordung des Hohepriesters Onias mit dem Tode bestraft wird (2Makk 4,38).

27 Für viele Anregungen und Gespräche zum Thema Münzen danke ich Siegfried Ostermann.

28 Vgl. HOWGEGO, Geld, 17.110-12.

29 So HÜBNER, Tradition, 171-87; WEISER, Massenmedium, 34-37. Eine ähnliche Position hat die Glyptik, vgl. hierzu SEIDL, Kultbilder, 89-114; NUNN, Bilder, 359-74. 
der die Zusammengehörigkeit und Identität in einem Reich gestärkt und die Herrschaft eines Königs präsentiert wird. Daher sind Münzen ein ideales Medium für die Selbstinszenierung eines Herrschers: ${ }^{30}$ Während auf der Vorderseite ein Porträt des Antiochus ${ }^{31}$ zu sehen ist, bietet die Rückseite der Münzen die Möglichkeit, seine Ansprüche darzustellen. ${ }^{32}$

Für die hier vorliegende Fragestellung sind drei Serien von Münzen interessant. ${ }^{33}$ Die älteste und wenig umfangreiche Münzemmision, deren Beginn um 175 anzusetzen ist, trägt auf der Rückseite lediglich die Aufschrift: „BA II $\Lambda \mathrm{E} \Omega \Sigma$ ANTIOXOY“ (König Antiochus). ${ }^{34}$ Dies ist die traditionelle Bezeichnung der seleukidischen Könige auf Münzen. Im Bild ist der auf einem Omphalos sitzende Apollon zu sehen.

30 Vgl. zum folgenden MørKHOLM, Studies; Le RIDER, Antioche; HOOver, Coins; BARAG, Mint, 59-77; Houghton, Coins; HOUGHTON/ LORBER, Coins; Bunge, Theos Epiphanes, 57-85.

31 „Die Herrscherdarstellungen auf den Münzen des Hellenismus sind nicht als ,Porträts' im modernen Sinn aufzufassen. Die Stempelschneider hatten keineswegs die Aufgabe, ein möglichst exaktes Abbild ihres Königs anzufertigen. Vielmehr stellen die Münzen dar, wie die Herrscher gesehen werden wollten. Sie sind daher mehr oder minder stark idealisiert, und auch scheinbar realistische Porträts sind mit $\mathrm{Ab}$ sicht in dieser Weise gestaltet". Vgl. EHLING, Untersuchung, 86, mit Verweis auf FLEISCHER, Studien, $1 \mathrm{ff} .81$.

32 Dass die Botschaft von Münzen in der Antike tatsächlich wahrgenommen und rezipiert wurde, lässt sich an der mitunter recht unmittelbaren Wirkung ablesen, wie z.B. die Nachahmung der Frisuren von römischen Kaisern auf den Münzen. So ZANKER, Power, 292-95. Auch wenn das Beispiel aus augustäischer Zeit stammt, ändert dies nichts an der Sache: Bilder wurden wahrgenommen und rezipiert. Die frühesten literarischen Hinweise auf gezielte Rezeption stammen aus dem 6./5. Jh. von dem Dichter Simonides (Fr. 114 Diehl = Diogenes Laertius 4.45), so HowGEGO, Geld, 84 .

33 Die Abfolge dieser drei Serien ist von MørKHOLM, Studies, 8-27, in die Jahre 175173/172, 173/172-169/168 sowie 169/168-164 datiert worden; ihm gefolgt ist LE RIDER, Antioche, 190-203. EHLING hingegen setzt die erste Serie auf 175/174, den Beginn der zweiten Serie bereits auf 174, weil Appian berichtet, dass Antiochus bereits bei seiner Regierungsübernahme von der antiochenischen Bevölkerung als „Epiphanes" begrüßt worden sei. Nach seinem Sieg über Ägypten (170-168 v.Chr.) hat Antiochus den Beinamen Nikephoros angenommen; weil aber die ersten Großbronzen ägyptischen Stils noch ohne den Beinamen Nikephoros geprägt wurden, setzt EHLING den Beginn der dritten Serie nach dem Tag von Eleusis, also nach Juli 168 an, EHLING, Untersuchung, 98; vgl. auch HOUGHTON/ LORBER, Coins, 61-62.

34 Münze Nr. 1395.2, Plate 5, in: Houghton/ LORBER, Coins, 63: “Obv. Diademed head of Antiochus IV r., diadem ends falling straight behind, fillet border; Rev. [...] Apollo seates 1. on omphalos, testing arrow and resting hand on grounded bow with grip marked by two pellets", datiert 175-173/2, Antiochia am Orontes. "The single tetradrachm of Series 1 from this die does not show the ends of the diadem. It thus remains uncertain whether this iconographic detail, symbolizing the king's apotheosis, was introduced with die A4 or added to the die at the start of the Series 2." 
Die zweite Münzserie, die kurz darauf, um 174 zu datieren ist, zeigt auf der Vorderseite Antiochus und auf der Rückseite erstmals Zeus mit einem Szepter und einer Figur auf seiner Hand. Dies ist die Personifikation des Sieges, die ihm den Siegeskranz überreicht. Es handelt sich hier also um den Typ des Zeus Nikephoros. Hier ist die Aufschrift er-

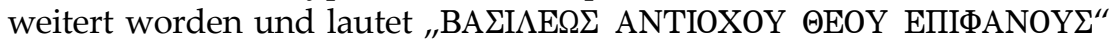
(König Antiochus erschienener Gott). ${ }^{35}$

Die dritte Serie ist nach dem 6. syrischen Krieg und dem Tag von Eleusis, nach Juli 168 v.Chr., emittiert. In ihr ist die Aufschrift noch einmal erweitert worden und lautet „BA $\mathrm{II} \Lambda \mathrm{E} \Omega \Sigma$ ANTIOXOY $\Theta E O Y$ ЕПІФАNOYऽ NIKНФОРОY" (König Antiochus erschienener siegreicher Gott). ${ }^{36}$ Interessant ist, dass nun die Figur mit ihrem Kranz das Wort „Epiphanes“ krönt. Zudem kann man ein sukzessives Wachstum der Beinamen beobachten.

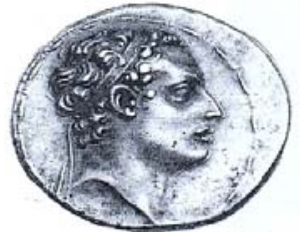

1395.2

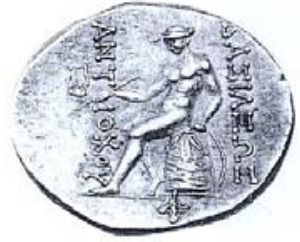

1. Serie: „BA $\Sigma \mathrm{I} \Lambda \mathrm{E} \Omega \Sigma$ ANTIOXOY"

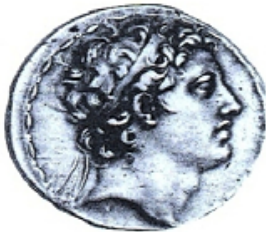

$1397 \mathrm{a}$

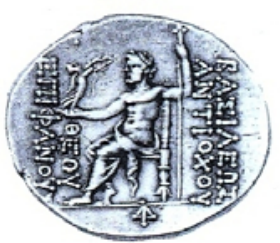

2. Serie: „BA $\mathrm{BI} \Lambda \mathrm{E} \Omega \Sigma \mathrm{AN}-$ TIOXOY ӨEOY ЕПIФANOY $\Sigma^{\prime \prime}$

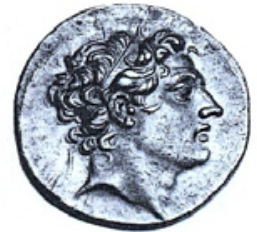

$1400 \mathrm{~d}$

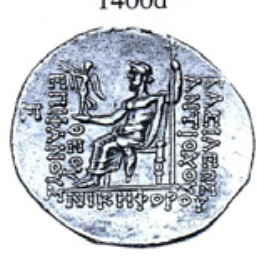

3. Serie: „BA $\Sigma \mathrm{I} \Lambda \mathrm{E} \Omega \Sigma$ ANTIOXOY $\Theta E O Y$ EПIФANOY N NIKHФOPOY“

35 Münze Nr. 1397, Plate 5, in: Houghton/ LORBER, Coins, 64: “Obv. Diademed head of Antiochus IV r., diadem ends adorned with stars, falling straight behind, fillet border; Rev. [...] Zeus enthroned 1., resting on scepter and holding Nike who faces r. and offers crown to Zeus, only one upright of throne back shown", datiert 173/2-169/8, Antiochia am Orontes.

36 Münze Nr. 1400d, Plate 5, in: Houghton/ LORBER, Coins, 64-65: “Obv. Diademed head of Antiochus IV r., diadem ends adorned with stars falling straight behind, fillet border; Rev. [...] Zeus enthroned 1., resting on scepter and holding Nike who crowns inscription", datiert 168-164, Antiochia am Orontes. 
Auch auf den neuen Bronzemünzen findet sich die Aufschrift „BA $\mathrm{BI} \Lambda \mathrm{E} \Omega \Sigma$

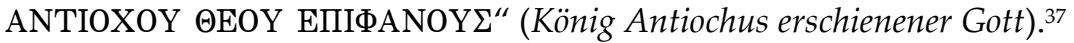

Antiochus IV. ist der erste seleukidische König, der auf den Münzen, die ihn auf der Vorderseite zeigen, seine Epitheta auf der Rückseite prägen ließ. ${ }^{38}$ Dies war im seleukidischen Reich neu, ist aber in der Folgezeit regelmäßig zu finden. Ebenso übernimmt Antiochus IV. als

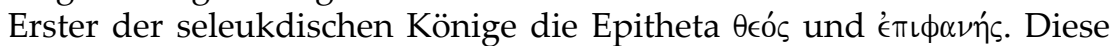
Epitheta werden einem Herrscher im Kontext des städtischen Herrscherkult aufgrund einer einmaligen, besonderen Leistung zugesprochen. ${ }^{39}$ So berichtet Appian, dass Antiochus IV. der Beiname „Epiphanes" von den "Syrern", vermutlich den Bewohnern Antiochias, angetragen worden sei, weil er sich als der rechtmäßige König erwiesen habe (Appian, Syriaca 45, 234).40 Damit könnte gemeint sein, dass Antiochus IV. nach dreizehnjähriger Geiselhaft in Rom und Athen im seleukidischen Reich wieder "erschienen“ sei und sich durch die Beseitigung des Königsmörders Heliodor als erfolgreicher König erwiesen

37 Münze Nr. 1438, Plate 64, in: Houghton/ LORBER, Coins, 78 vgl. 77: “Obv. Radiate, diademed head of Antiochus IV r., one diadem end flying up behind, the other falling forward over shoulder, dotted(?) border; Rev. Zeus seated 1., on high-backed throne, holding phiale(?) and resting on sceptre", wahrscheinlich Dura Europos, datiert um $173 / 2$.

38 Die Rückseite setzen sich aus dem Königstitel, dem Namen des Königs und bis zu drei Kultnamen zusammen. Die offiziellen Epitheta lassen sich in dynastische (Eupator, Theopator, Philadelphos etc.), in religiös-kultische (Epiphanes, Eusebes, Theos Epiphanes etc.) und politisch-militärische Beinamen (Euergetes, Megas Euergetes, Nikephoros, Soter etc.) einteilen, die wichtige Hinweise auf die Legitimationsgrundlagen und das charismatische Selbstverständnis des Königs geben. Die Grenze zwischen der zweiten und dritten Gruppe ist fließend; viele Könige führen Beinamen aus verschiedenen Kategorien, vgl. hierzu EHLING, Untersuchung, 97.

39 Die auf individueller Tatkraft und sichtbarer Leistung (' rende Herrschaft des Königs kommt in den Epitheta, die den Königen gegeben wer-

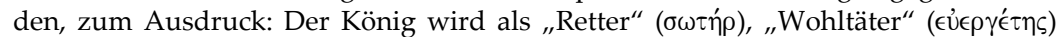

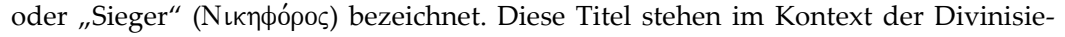
rung der Herrscher und fungieren als deren Kultnamen, die diesen aufgrund besonderer Leistung, z.B. der Rettung einer Stadt, zukamen und die diese dann in theologischen Kategorien interpretieren: Der siegreiche König ist der, der sein Volk rettet, den Sieg bringt und sich als Wohltäter erweist.

40 Es scheint üblich gewesen zu sein, dass Kultnamen den Königen von der Bevölkerung verliehen wurden. So ist z.B. Demetrios I. von den Bewohnern der Stadt Babylon als $\sigma \omega \tau \eta \dot{\rho} \rho$ „Retter“ begrüßt worden, als er den abtrünnigen Satrapen Timarchos besiegt hatte; vgl. Appian, Syriaca 47, 242; 67, 353. Appian lässt übrigens die auf den Münzen belegte Bezeichnung $\theta \epsilon o ́ c$ unerwähnt, was daran liegen kann, dass bei

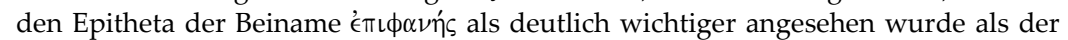
Titel $\theta \epsilon o ́ c$. Allerdings ist es auch möglich, dass Antiochus IV. den Titel $\theta \epsilon o ́ c$ zu dem

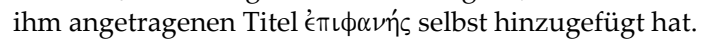


habe. Die für eine einmalige, besondere Tat zugesprochenen Epitheta übernimmt Antiochus aber als dauerhafte Beinamen und lässt sie als erster seleukidischer König auf Münzen prägen. Mit dieser offiziellen Verwendung der Epitheta verändert sich der Charakter der Beinamen: „Durch die Kombination mit theos verliert das Epitheton epiphanes seinen Bezug auf die Ereignisse des Jahres 175 und damit deutlich die Konnotation einer einmaligen, rettenden Tat, es wird vielmehr zum Dauerzustand. Er war nicht bloß als besonderer Mensch und Retter in einer politisch brisanten Situation erschienen, sondern um dauerhaft auf Erden positiv zu wirken. “41 Über diesen Vorgang ist viel spekuliert worden: Während seit Bevan v.a. theologische Gründe angeführt werden, ${ }^{42}$ kann Bunge zeigen, dass es handfeste politische Gründe für die Übernahme dieses theologischen Kultnamens gab:43 Die Provinz Koilesyrien und Phönizien war 200 v.Chr. durch den Vater von Antiochus IV., Antiochus III., der ptolemäischen Herrschaft entrissen worden. Um die politischen Verhältnisse zu stabilisieren und Einfluss auf die ptolemäische Erbfolge zu nehmen, war die Verbindung der beiden Königshäuser durch die Heirat von König Ptolemaios V. (197-181/180 v.Chr.) mit Kleopatra, der Tochter von Antiochus III., verstärkt worden. Auf ptolemäischer Seite war es Ptolemaios V., der als Erster den Beinamen "Epiphanes" dauerhaft annahm. Anfang der 170er Jahre wurden das ptolemäische wie das seleukidische Reich von noch unmündigen Königen regiert: Antiochus Seleukou im seleukidischen und Ptolemaios VI. im ptolemäischen Reich; ersterer ist der Stiefsohn, letzterer der Neffe von Antiochus IV. Bunge vermutet, dass Antiochus IV.

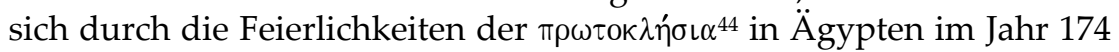
v.Chr. herausgefordert sah, als Ptolemaios VI. den Beinnamen ФI $\Lambda$ OMHT $\Omega$ P angenommen hatte, was Antiochus so verstanden zu haben scheint, dass Ptolemaios VI. damit seine Ansprüche auf die Provinz Koilesyrien und Phönizien über die Abstammung seiner seleukidischen Mutter in der Öffentlichkeit propagiert habe. Daraufhin habe Antiochus IV. seinerseits mit der Übernahme des Beinamens "Epiphanes" reagiert, den erstmals der Vater des amtierenden ptolemäischen Königs Ptolemaios VI. angenommen hatte. Diesen Beinamen habe er sogleich auf internationaler Ebene durch die Tetradrachmen publik gemacht und entsprechend propagiert. Wie er als Onkel und Stiefvater die Herrschaft (wenn auch nominell) für seinen minderjährigen Neffen Antio-

41 MitTAG, Antiochos IV. Epiphanes, 129-30.

42 Vgl. Bevan, Note, 26.

43 Vgl. Bunge, Theos Epiphanes, 68-83. Vgl. auch BunGE, Religionsverfolgung, 155-65.

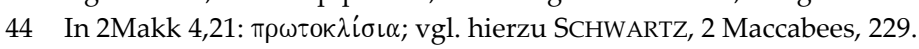


chus Seleukou übernommen hatte, so könnte er durch die Übernahme des Beinamens „Epiphanes“, den ja Ptolemaios V. als erster angenommen hatte, seine Ansprüche gegenüber Ptolemaios VI., zu dem er ja als Onkel im gleichen verwandtschaftlichen Verhältnis stand, deutlich gemacht haben. Zugleich markiert Antiochus IV. mit den Münzen seine Vorherrschaft gegenüber seinem Neffen Antiochus, von dem von diesem Zeitpunkt an so gut wie keine Münzen mehr geprägt wurden.

$\mathrm{Ab}$ 173/172 finden sich weitere Darstellungen bzw. Epitheta auf den

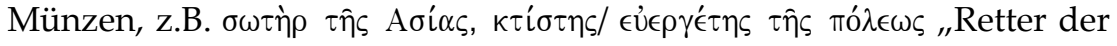
(Provinz) Asia, Gründer/ Wohltäter der Stadt" ${ }^{\prime 4}$

Auf seleukidischen Tetradrachmen ist zudem der Wechsel von dem Standardtyp des auf dem Omphalos sitzenden Apoll zu dem thronenden Zeus mit Nikestatue zu beobachten. Apoll war die Schutzgottheit der Seleukiden, die sich auf den Licht- und Heilgott Apoll als ihren Stammvater zurückführen. ${ }^{46}$ Zeus Nikephoros scheint Antiochus IV. im Jahre 174 eingeführt zu haben. ${ }^{47}$ Dabei scheint sich Antiochus IV., anders als häufig vermutet wird, keineswegs mit Zeus Olympios identifiziert zu haben, vielmehr liegen auch hier die Gründe auf politischer Ebene: Apoll galt seit dem Begründer der Dynastie, Seleukos I., als Schutzgott des Königshauses und dessen göttlicher Vater. Rechtmäßiger König aber war Antiochus Seleukou, nicht Antiochus IV. Es könnte sein, dass Antiochus IV. nun seinerseits seine göttliche Verbindung aufzuweisen suchte, indem er sich als „erschienener Gott“ präsentierte. Trotz der intensiven Selbststilisierung von Antiochus IV. mit göttlichen Attributen auf den Münzen kann zu seiner Zeit jedoch keine gezielte Religionspolitik nachgewiesen werden, die der Propagierung und Durchsetzung des Zeus-Kults gedient hätte. ${ }^{48}$

Aber auch die Porträts auf der Vorderseite haben ebenfalls programmatischen Charakter und bieten Raum für unterschiedliche Inszenierungen: So hat Antiochus IV. als erster Seleukidenkönig sich auf den Münzen als Alexander darstellen lassen. ${ }^{49}$ Zugleich haben die Porträts die Funktion, den Porträtierten an eine Gottheit anzugleichen: Daher finden sich im ikonographischen Programm der Antiochus-Münzen an

45 Die Denkfigur der „Epiphanie“ zeigt sich auch in den Stadtumbenennungen: So erhielten zwei bis max. zehn Städte von Antiochus IV. den Namen "Antiocheia", andere aber nannte er auch in „Epiphaneia“ um. Vgl. zur kritischen Diskussion der Belege MitTAG, Antiochos, 201-08.318.

46 Vgl. hierzu EHLING, Untersuchung, 90-92.

47 Daher wird meist der Zeus Nikephoros-Typ als die jüngere auf Antiochus IV. zurückgehende Linie gegenüber der älteren Apollonlinie verstanden.

48 Vgl. hierzu MiTTAG, Antiochos, 139-45, mit Verweis auf entsprechende Literatur.

49 So EHLING, Untersuchung, 86-88. 
den Enden des Königsdiadems Sterne bzw. ein Stern ${ }^{50}$ oder Antiochus wird im Strahlenkranz bzw. mit einem Stern oberhalb der Stirn gezeigt. ${ }^{51}$ Mit diesen Darstellungen wird die Gottähnlichkeit oder Göttlichkeit von Antiochus IV. im Sinne der "Metapher der strahlenden Epiphanie ${ }^{\prime 52}$ dargestellt. Sterne und Strahlenkrone zeigen die besondere Verbindung zwischen dem König und der göttlichen Sphäre an. So ist Antiochus der erste Seleukidenkönig, der sich mit Strahlenaureole oder Strahlendiadem an Helios angleicht. ${ }^{33}$

165 bricht Antiochus IV. in die östlichen Herrschaftsgebiete auf und beabsichtigt, Armenien, das sich nach dem Tod von Antiochus III. selbständig gemacht hatte, zurück zu erobern. Über seine anabasis ist nicht viel bekannt, doch scheint Antiochus beabsichtigt zu haben, wirtschaftliche Impulse v.a. am Persischen Golf zu setzen, internationale Handelsrouten zu fördern und den Fernhandel mit Indien und China, besonders im Hinblick auf die mangelnden eigenen Metallressourcen, zu stärken. Ebenso ist überliefert, dass Antiochus IV. versucht haben soll, den Artemis-Tempel von Elymais zu plündern. Die unterschiedlichen Berichte $^{54}$ über Tempelplünderungen ergeben jedoch kein einheitliches Bild, sondern können - zumindest in ihrer Ausgestaltung - propagandistische Ausschmückungen gegen Antiochus IV. sein. Denn auffallend ist, dass in den Schilderungen immer wieder Geldnot als Motiv für seine anabasis angegeben wird. Da die östlichen Gebiete politisch v.a. um einen Tempel organisiert sind, erfolgt die Zahlung der Abgaben und Steuern an die Seleukiden über die mit dem Tempel verbundene lokale Führungselite. Diese haben bei einem schwachen seleukidischen König die geforderten Abgaben im eigenen Sinne oft nach unten korrigiert. Fordert nun der seleukidische König, gestützt auf seine militärische Präsenz vor Ort, die noch ausstehenden Zahlungen ein, so tut er dies aus seiner Sicht durchaus zu Recht. Dies bedeutet für die lokalen Eliten, dass sie die Steuerschulden schnell eintreiben müssen und unter Umständen auch auf die eigenen Reserven, die im Tempel verwaltet werden, zurückgreifen müssen. Vor diesem Hintergrund kann das, was aus seleukidischer Perspektive das rechtmäßige Einfordern von säumi-

50 Vgl. Münze Nr. 1397a oder 1474, in: Houghton/ LORBER, Coins, bzw. die Abbildungen bei MITTAG, Antiochos, 132.

51 Vgl. Münze Nr. 1474, in: HOUGHTON/ LORBER, Coins.

52 KYRIELEIS, Sternsymbolik, 67.

53 So EHLING, Untersuchung, 86-88.

54 Polybios 31.9; 1Makk 3,31.37; 6,1-3; 2Makk 1,13-17; 9,1-2; A.J. 12.354-355; Appian, Syriaca 352; Porphyrius, Fragmente der griechischen Historiker 260 F 53; 56,1; Hieronymus, Expl. Dan. 11.36. 
gen Steuerzahlungen ist, ${ }^{55}$ in lokaler Perspektive als Tempelplünderung erscheinen. Weil Judäa ähnlich strukturiert ist, ist die Heliodor-Erzählung (2Makk 3) vor diesem Hintergrund zu verstehen. ${ }^{56}$

Im November/ Dezember 164 stirbt Antiochus IV. überraschend während seiner anabasis. Sterbeort und Todesursache sind unbekannt. ${ }^{57}$ Viele Quellen machen die „Tempelplünderungen“ für seinen Tod verantwortlich. So schildert das Zweite Makkabäerbuch im neunten Kapitel eine eigene Version von Antiochus' Tod. ${ }^{58}$

\section{Das Epiphanie-Motiv im Zweiten Makkabäerbuch: Antiochus Epiphanes und der epiphane Gott}

Im Zweiten Makkabäerbuch finden sich zwei unterschiedliche „Epiphanie“-Linien: zunächst wird das Epitheton „Epiphanes“ des Königs Antiochus aufgegriffen und an herausgehobenen Stellen im Zweiten Makkabäerbuch verwendet: im Vorwort (2Makk 2,20), bei seinem ersten Auftritt (2Makk 4,7) und bei seinem Tod (2Makk 10,9.13). Darüber hinaus gibt es sechs, im Vorwort angekündigten Epiphanie-Schilderungen (2Makk 2,21; 3,24-26.31-34; 5,1-4; 10,29-30; 11,8; 12,22; 15,1116.25-36). Somit stellt sich das mit dem Lexem $€ \pi\llcorner\phi \alpha \nu-59$ verbundene Motivfeld als ein bedeutendes "Schlüsselwort"60 der Erzählung dar. Unabhängig von der Frage, was die historische Person Antiochus IV. selbst unter seinem Beinamen „Epiphanes" verstanden haben mag, ${ }^{61}$ ist diese literarische Komposition des Zweiten Makkabäerbuches kein Zu-

55 Vgl. hierzu auch in Bezug auf 2Makk 3 FISCHER, Heliodor, 121-38. Vgl. hierzu auch BRINGMANN, Geschichte, 103.

56 Vgl. hierzu BICKERMANN, Héliodor, 7-40; wieder abgedruckt in erweiterter Fassung in: BICKERMANN, Studies, 161-66

57 Vgl. MitTAG, Antiochos, 319.328-31.

58 Weitere Erzählungen über Antiochus' Tod finden sich in 1Makk 6,1-16; 2Makk 1,1317; Polybios 31.9; Josephus, A.J. 12.354-350; Diod. 31.18a; Appian, Syriaca 352 u.a.

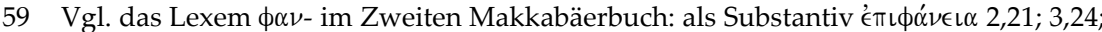

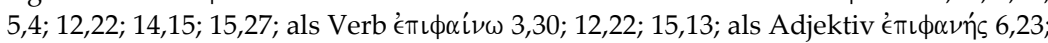
14,33; 15,34; als Simplex $\phi \alpha i v \omega 1$ 1,33; 3,25.33; 5,2; 6,27; 7,22; 10,29; 11,8; 12,9.16.36;

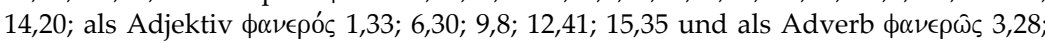

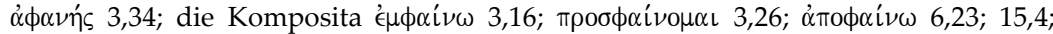

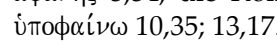

60 So HABICHT, 2. Makkabäerbuch, 187.

61 "There is little evidence to suggest that Antiochus attached much importance to his divine status or used it to unite his empire in worship of him. The title was often simply 'Epiphanes', which may mean little more than 'Illustrious'“, BARTLETT, Book, 21-22. 
fall. Wie wird nun das Epiphaniemotiv im Zweiten Makkabäerbuch entfaltet?

Die Epiphanieerzählungen des Zweiten Makkabäerbuches dienen dazu, mit Hilfe der Epiphanien ein Porträt des Gottes Israels zu zeichnen, um mit diesem ein Gegenbild zur Figur des Königs Antiochus Epiphanes zu entwerfen. Schließlich ist - aus der Perspektive der Erzählung - der Gott Israels durch den Versuch der Umwidmung des Jerusalemer Tempels in einen Kultort für Zeus Olympios radikal in Frage gestellt worden (2Makk 4; 6). Da aber eine direkte Konfrontation für den transzendent gedachten Gott Israels unvorstellbar ist, wird die Auseinandersetzung zwischen Gott und Antiochus mit den Mitteln der Epiphanie und der mit ihr verbundenen Motive dargestellt.

Interessant ist zunächst die Verteilung der Epiphanien in der Erzählung: Die großen Epiphanien finden unter Vorgängern und Nachfolgern von Antiochus statt. Die einzige Epiphanie, die in die Zeit der Herrschaft des Antiochus Epiphanes fällt, ist die „eher ominöse“62 und schwer zu interpretierende zweite Epiphanie (2Makk 5,1-4), bei der of-

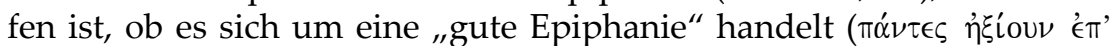

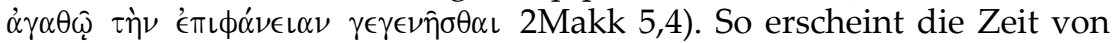
Antiochus Epiphanes als eine für Israel epiphaniearme Zeit. ${ }^{63}$ Dadurch stellt sich die Frage nach der Mächtigkeit Gottes angesichts des Königs Antiochus IV. umso schärfer, was exemplarisch an der ersten und letzten Epiphanie erläutert werden soll:

Die erste Epiphanieschilderung findet noch vor Antiochus' Regierungsantritt statt (2Makk 3,24-26.31-34) und bewirkt die Rettung des Jerusalemer Tempelvermögens vor Heliodor. Durch die erste Epiphanie werden in Jerusalem Furcht und Schrecken durch die sichtbare Erscheinung des allmächtigen Herrn in Freude und Herrlichkeit verwan-

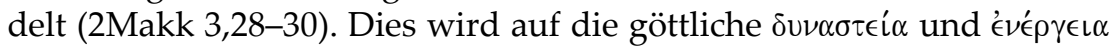
zurückgeführt (vgl. 2Makk 3,28-30). Ein ähnlicher emotionalen Umschwung zeigt sich auch in der letzten Epiphanie (2Makk 15,11-16.2536), in der Judas und seinem Gefolge ihren völlig unerwarteten Sieg gegen das Heer des Nikanor erlangen. Dieser Sieg wird als „Epiphanie Gottes“ wahrgenommen (2Makk 15,27), durch den Furcht und Zittern

62 So FRENSCHKOWSKI, Offenbarung, 106.

63 Dies mag aber auch damit zusammenhängen, dass, abgesehen von der ersten, die in der noch idealen Zeit unter dem rechtmäßigen Hohepriester Onias spielt (2Makk 3,24-26.31-34), die Epiphanien die unterlegenen jüdischen Truppen im Kampf militärisch unterstützen (2Makk 10,29-30; 11,8; 12,22; 15,11-16.25-36). Diese jüdischen Truppen haben sich aber erst nach bzw. im Kontext der Verfolgung von Antiochus gebildet (2Makk 8), Antiochus IV. aber stirbt bereits kurz darauf (2Makk 9), vgl. hierzu auch VAN HENTEN, Selbstverständnis, 135-36. 
(2Makk 15,23) in Freude verwandelt werden (2Makk 15,27.28). Das Ergebnis dieser Epiphane ist: „Alle aber insgesamt rühmten, zum Him-

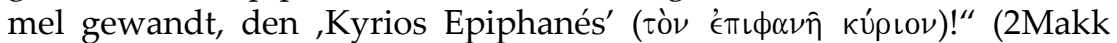
15,34). ${ }^{64}$ Damit endet die Erzählung, die mit Antiochus IV., dem Epiphanes, begonnen hat, mit dem Gott Israels, als dem Kyrios Epiphanes.

So ergibt sich, dass sich die epiphaniereiche Erzählung des Zweiten Makkabäerbuches offensichtlich am Beinamen Antiochus' IV. inspiriert hat. Die Epiphanieschilderungen werden damit nicht (nur) um ihrer selbst willen erzählt, sondern sind (auch) Gegenerzählungen gegen Antiochus, der sich als „Epiphanes“ bezeichnet. Dass bereits zur Zeit von Antiochus sein Beinamen „Epiphanes“ Anlass für Wortspiele war, zeigt sich in der Überlieferung, dass man Antiochus statt "Epiphanes" als „Epimanes“ („Wahnsinniger") bezeichnet habe (vgl. Polybios 26.1).

Während die Selbstinszenierung des seleukidischen Königs Antiochus als „Epiphanes“ das Epiphaniemotiv im Zweiten Makkabäerbuch inspiriert haben dürfte, stellt sich die Frage, welche Auswirkungen dies für das Porträt des Gottes Israels in der Erzählung hat.

Während der Gott Israels im Zweiten Makkabäerbuch durchge-

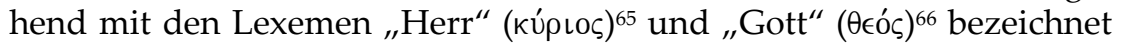
wird, ${ }^{67}$ werden in der Erzählung außerdem eine Reihe weiterer Gottesbezeichnungen verwendet. ${ }^{68}$ Alle diese Gottesbezeichnungen betonen die Souveränität und Allmacht Gottes, so z.B. der Begriff „Pantokrator"

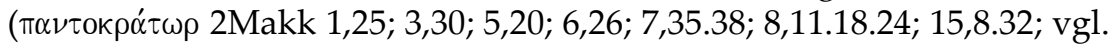

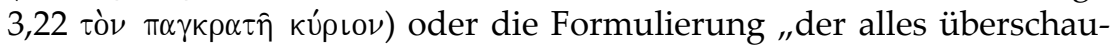

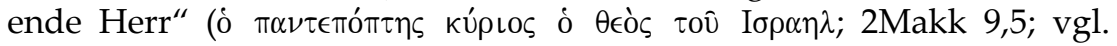

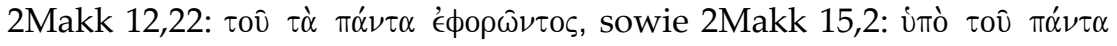

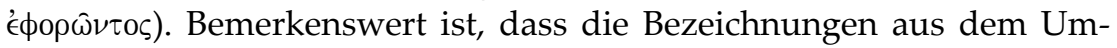
feld der Königsterminologie stammen: So wird der Gott Israels mit dem persischen Königstitel als der „König der Könige“ (ó $\beta \alpha \sigma \iota \lambda \in \dot{\nu} \varsigma \tau \hat{\omega} \nu$

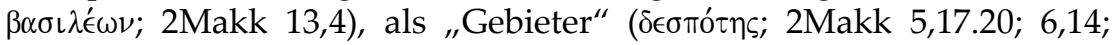

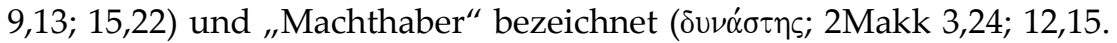

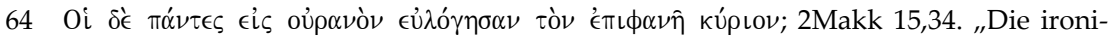
sche Hindeutung auf Antiochus IV. Epiphanes ist offensichtlich“ (BÉNEVOT, Makkabäerbücher, 247).

65 Kúpıos in 2Makk 1,8.24; 2,2.8.10.22; 3,22.30.33.35; 4,38; 5,19; 6,30; 7,6.20.33.40; 8,2.5. 14.27.29.35; 9,5; 10,1.4.28.38; 11,6.10; $12,36.41 ; 13,10.12 .17 ; 14,35.36 ; 15,4.7 .21 .34$.

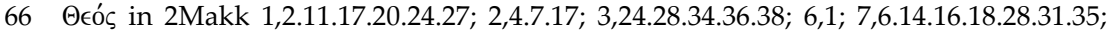
8,13.18.23; 9,5.8.12.17; 10,16.25; 11,4.9.13.23; 12,6.11.16; 13,13.15; 14,33; 15,14.16.27.

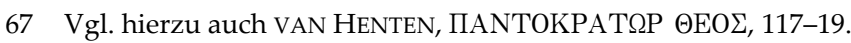

68 Zur Geschichte und Entwicklung dieser Gottesbezeichnungen vgl. ZIMMERMANN, Namen. 


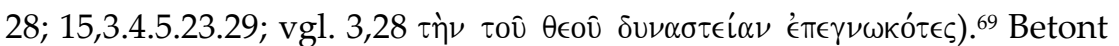

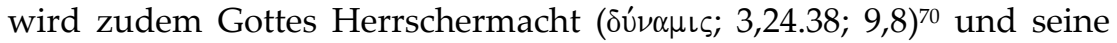

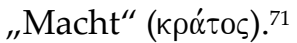

Dass der Gott Israels in dieser expliziten Weise mit Herrschaftsterminologie verbunden wird, ${ }^{72}$ geschieht im Zweiten Makkabäerbuch nicht nur mit der alttestamentlich vertrauten Redeweise von Gott als dem „König“, sondern weist auch ein deutlich eigenes Profil auf: So ist mit Gott das Motivfeld des Kampfes, des Sieges und der Hilfe verbun-

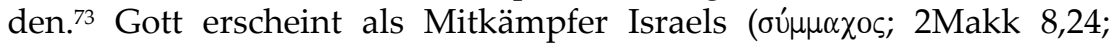
10,$17 ; 11,13$ ), als derjenige, der für die Juden kämpft (ímé $\rho \mu \alpha \chi 0 \varsigma ; 2 M a k k$ 8,36; 14,34) und der Israel in den Epiphanien himmlische Streiter zur

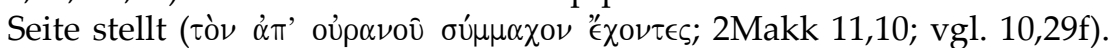
Von daher verwundert es nicht, dass die beiden Kampfparolen

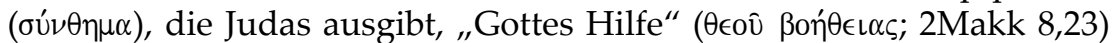

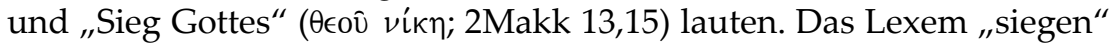
$(\nu$ เK-) ist (mit Ausnahmen von 2Makk 3,5 und 8,33) ausschließlich mit Gott verbunden und findet sich vor allem am Ende der Erzählung: Siegreich sind die jüdischen Truppen nur durch ihr Vertrauen auf Gott (2Makk 10,28), dass er ihnen den Sieg schenkt (2Makk 10,38). Weil Gott an ihrer Seite ist, sind sie unbesiegbar (2Makk 11,13). Im letzten Kampf erinnert Judas seine Leute an Gottes Taten in der Geschichte, die auf einen Sieg hoffen lassen, den sie vom Allherrscher erwarten dürfen (2Makk 15,8): Und dieser stellt sich dann tatsächlich ein (2Makk 15,26f). ${ }^{74}$ Was bedeutet das nun? Durch die Sieg-Metaphorik wird im Zweiten Makkabäerbuch der Gott Israels wie ein hellenistischer König vorgestellt, dessen wichtigstes Kennzeichen seine Sieghaftigkeit ist:75 Gerade die letzte Erzählung über den Kampf gegen Nikanor macht ex-

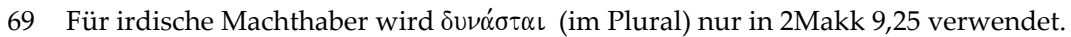

$70 \Delta$ úv $\mu \iota \varsigma$ wird in der Bedeutung "Streitmacht“ in 2Makk 1,13; 10,24; 13,2 verwendet.

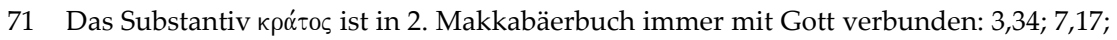
9,$17 ; 11,4 ; 12,28$.

72 Die drei für das Gottesverständnis zentralen Wörter und Wendungen finden sich gleich in der ersten Epiphanie-Schilderung: Dies ist erstens die Rede von Gott als

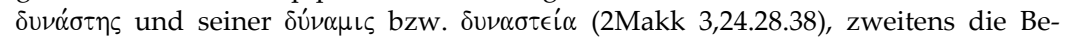

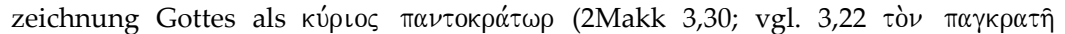

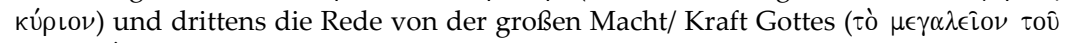

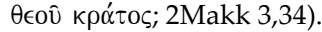

73 Das Lexem ßoך $\theta$ - findet sich in 2 Makk 3,39; 6,11; 8,20.35; 12,11; 13,10.13; 15,8.35 für den Gott Israels, darüber hinaus noch in 2Makk 3,28; 6,11; 8,8; 9,2; 11,7.

74 Dass das Lexem $\nu$ เк- gehäuft im Kontext der Auseinandersetzung mit Nikanor auf-

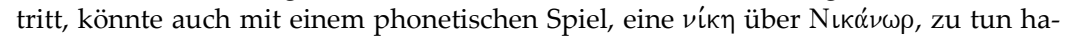
ben.

75 Vgl. hierzu auch die Artikel in dem Sammelband von RAJAK, Perspectives. 
plizit deutlich, dass Judas mit seinen Leuten derart unterlegen ist, dass ein Sieg militärisch völlig unwahrscheinlich ist (2Makk 15,21). Judas siegt, was aber allein auf Gottes helfendes Eingreifen in der letzten Epiphanie zurückzuführen ist, der sich in dieser Auseinandersetzung "epiphan" zeigt (2Makk 15,25-29.34).

Dass der Gott Israels im Zweiten Makkabäerbuch nach dem Typus eines hellenistischen Herrschers porträtiert wird, zeigt sich auch an weiteren Begriffen der hellenistischen Herrscherideologie, die auf den Gott Israels übertragen werden: So wird Heliodors Zusammenbruch im

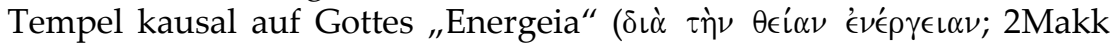
3,29) zurückgeführt.76 Er erscheint als derjenige, der „Rettung“ bringt ( $\sigma \omega \tau \eta \rho i ́ \alpha ; 2$ Makk 11,6) und den man als "Retter" preist und bekennt

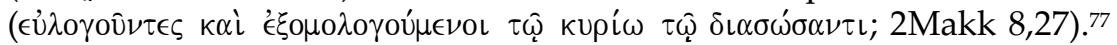

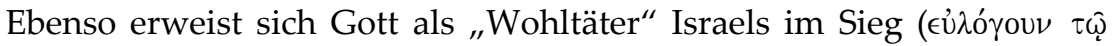

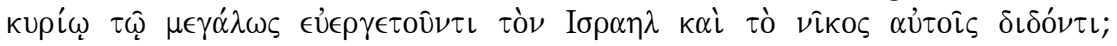

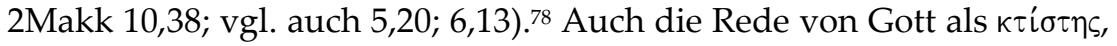
die nach einer Rede von Gott als "Schöpfer" klingt, ist vor dem Hintergrund hellenistischer Königsideologie eher Rede von Gott als dem "Gründer", der nicht nur Städte gegründet oder umbenannt hat, son-

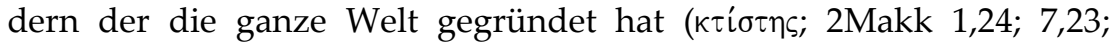
$13,14){ }^{79}$

Im Zweiten Makkabäerbuch wird der Gott Israels somit im Typus eines hellenistischen Herrschers charakterisiert, der aber - anders als diese - nicht selbst und persönlich die Kämpfe anzuführen und durchzufechten hat, sondern der seine Präsenz vermittelt in den unterschiedlichen Epiphanien sichtbar zum Ausdruck bringt. Statt dass sich Gott selbst in prächtiger Rüstung und blinkenden Waffen zeigt, wie dies hellenistische Könige tun, ${ }^{80}$ werden nur die in den Epiphanien auftretenden göttlichen Mittlergestalten als solche Kämpfer dargestellt. Liest man das Zweite Makkabäerbuch vor diesem Hintergrund, dann eröffnet sich eine neue hermeneutische Perspektive auf die so ungehemmt geschilderte Gewalt in der Erzählung: Bei der Porträtierung Gottes als

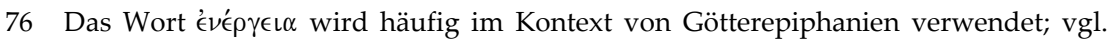
NILSSON, Geschichte, 215.

77 Darüber hinaus findet sich „Rettung" ( $\sigma \omega \tau \eta \rho i ́ \alpha)$ in 2Makk 3,29.32; 7,25; 12,25; 13,3; 14,3 (vgl. 2Makk 1,11;2,17).

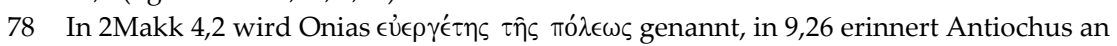
seine $\epsilon \dot{U} \in \rho \gamma \epsilon \sigma i \alpha \iota$ an den Juden.

79 Vgl. hierzu auch SCHMITZ, Nichts, 199-216.

80 So etwa auch Antiochus bei seinen 30-tägigen Feierlichkeiten und der alle Dimensionen sprengenden Parade in Daphne 166 v.Chr.; vgl. hierzu MITTAG, Antiochos, 282-95. 
einen idealen hellenistischen Herrscher geht es in keiner Weise darum, Gewalt durch Gott zu legitimieren, zu rechtfertigen oder gar zu verherrlichen, vielmehr sind die ausführlichen Kriegsschilderungen ein literarisches Mittel in hellenistischer Zeit, um durch die Sieghaftigkeit zu erzählen, dass Gott sein König-Sein erfolgreich, sichtbar und epiphan zeigt.

Mit der Charakterisierung Gottes als idealen hellenistischen Herrscher eröffnet sich in der Konzeption des Zweiten Makkabäerbuches eine doppelte Frontstellung: zum einen gegenüber Antiochus Epiphanes, zum anderen gegenüber dem Makkabäer Judas.

Die erste wird im Martyrium der sieben Brüder von dem sechsten Bruder gegenüber Antiochus auf den Punk gebracht: Er wirft Antiochus vor, gegen Gott zu kämpfen $(\theta \in о \mu \alpha \chi \epsilon \in \omega ; 2 M a k k ~ 7,19)$ und benennt damit den eigentlichen Konflikt: Antiochus, der sich mit seinem Bei-

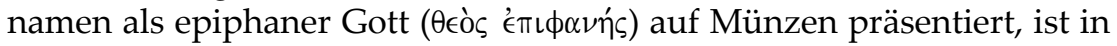

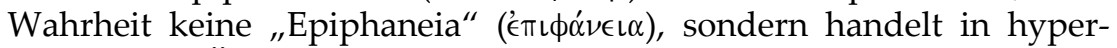
ephania, in „Überheblichkeit" und maßloser Selbstüberschätzung (i்tep-

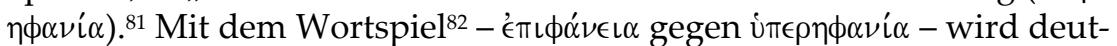
lich, dass Antiochus kein Gott ( $\theta \in o ́ \varsigma)$, sondern gottlos ist (2Makk 10,10). Bereits der fünfte Bruder hat betont, dass Antiochus nichts als ein

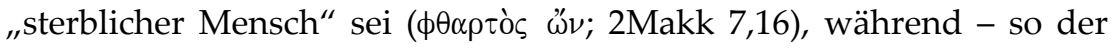
sechste Bruder - der Gott Israels der einzige Gott sei (2Makk 7,37). Zu dieser Erkenntnis kommt Antiochus selbst aber erst in seinem qualvollen Tod, in dem er erkennt, dass er selbst nur ein sterblicher Mensch

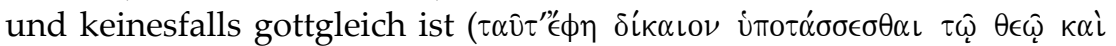

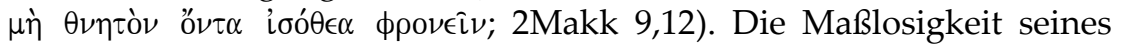
Anspruchs, ein epiphaner Gott zu sein, wird somit in der Erzählung widerlegt. Anders als er, der sich gerade nicht „nachsichtig und men-

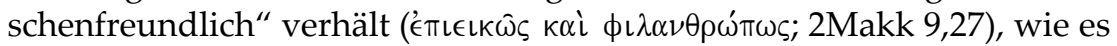
sich für einen guten König gehören würde, ist es der Gott Israels, der sich Israel in seinem Wohlwollen zeigt (

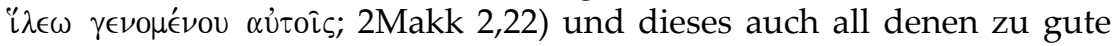
kommen lässt, die seine Macht anerkennen. So erweist Gott sich z.B. für Heliodor als Lebensretter und Wohltäter, als guter König. Auf diese Weise ergibt sich ein Bogen von der ersten Epiphanie-Erzählung, die

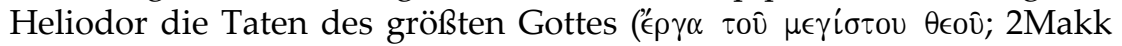

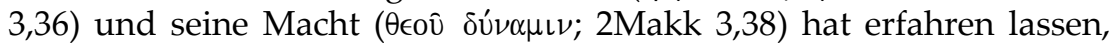
zur Gotteserkenntnis Israels, das Gott als den Epiphanen erlebt (2Makk

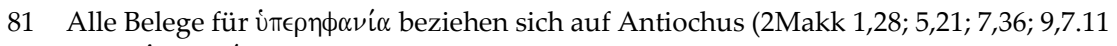

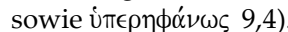

82 So auch SCHWARTZ, 2 Maccabees, 81.355. 
15,27.34). Wie es sich für einen guten hellenistischen Herrscher gehört, wird die erzählte Sieghaftigkeit Gottes im Zweiten Makkabäerbuch in zwei großen Festen gefeiert, die bleibend an den Sieg Gottes in seinem "Zweikampf" gegen Antiochus und gegen Nikanor erinnern (2Makk $10,8 ; 15,36)$.

Neben der Kritik an Antiochus Epiphanes findet sich aber noch eine zweite, wenn auch etwas verstecktere, königskritische Linie: Während Gott selbst im Zweiten Makkabäerbuch nicht handelnd auftritt, fungiert Judas als derjenige, der handelt. Er hat eine herausgehobene Stellung, seine Brüder hingegen werden nur am Rande und nur sehr kritisch erwähnt. Gerade im Vergleich mit dem so hasmonäerfreundlichen Ersten Makkabäerbuch ist die Zurückhaltung des Zweiten Makkabäerbuches gegenüber den Hasmonäern auffallend. ${ }^{33}$ Aber auch gegenüber Judas ist das Zweite Makkabäerbuch kritisch eingestellt: Ohne Gottes Hilfe hätte Judas keinen militärischen Erfolg gehabt und keinen Sieg errungen (vgl. 2Makk 8,2-5.27; 13,17; 15,21). Vor diesem Hintergrund wäre zu fragen, ob sich nicht eine vorsichtig-kritische Haltung gegenüber der Königsherrschaft der Hasmonäer im Zweiten Makkabäerbuch findet, die den ersten „Hasmonäer" Judas deshalb lobt, weil er militärisch nicht erfolgreich war, sondern den Sieg nur durch den wahren und sich epiphan erweisenden König Gott errungen hat. So erzählt das Zweite Makkabäerbuch das (bedingte) Lob des ersten Makkabäers Judas gegenüber den späteren Hasmonäern, die sich in kürzester Zeit aus den gegen die Hellenisierung kämpfenden Makkabäern zu hellenistischen Machthabern entwickelt haben. ${ }^{84}$

\section{Fazit}

Das Zweite Makkabäerbuch ist weit mehr als eine Erzählung, die nur

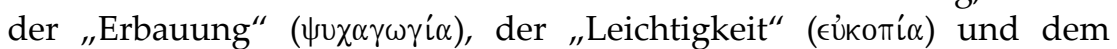
"Nutzen" ( $\left.\dot{\omega}^{\prime} \dot{\epsilon} \lambda \epsilon \iota \alpha\right)$ dient (2Makk 2,25). Die Gefühle und Affekte, die es hervorrufen will, gehen darüber hinaus, denn Vergnügen und Leichtigkeit sind Vehikel, über die die Leserinnen und Leser gefesselt werden sollen, um zu Nutzen und zu nützlicher Kenntnis zu gelangen.

83 So wird Modeïn nur einmal in 2Makk 13,4 als Lagerort für Judas' Heer genannt; Judas' Bruder Simeon (Vater von Johannes Hyrkan) wird nur einmal und zwar sehr kritisch erwähnt (2Makk 10,19-22; 14,17); die Heldentat eines anderen Judas-Bruders Eleasar (1Makk 6,43-46) wird in 2Makk 13,15 Judas zugeschrieben, obwohl der Verfasser die Namen der Brüder kennt (2Makk 8,22-23); vgl. hierzu ENGEL, Bücher, 327, und HABICHT, 2. Makkabäerbuch, 188.

84 Zum historischen Kontext vgl. auch SCHWARTZ, Maccabees, 29-40. 
Damit sind Emotionen, Gefühle und Affekte keineswegs nur Inhalt und Gegenstand der Erzählung, wie z.B. in den Martyriumsschilderungen, die die Leserinnen und Leser sicherlich mit Schaudern und Entsetzen lesen. Emotionen, Gefühle und Affekte sind die Kommunikationsabsicht der Erzählung, deren Ziel die $\omega \dot{\phi} \dot{\epsilon}^{\prime} \lambda \epsilon \iota \alpha$, die nützliche Kenntnis, ist: Die Leserinnen und Leser sollen zu ihrem Nutzen lernen. Was aber sollen sie lernen?

Das Zweite Makkabäerbuch inspiriert sich offensichtlich an der medialen Selbstinszenierungen und dem epiphanen Gottesanspruch des seleukidischen Königs Antiochus, wie er historisch exemplarisch an den Münzen greifbar ist. Mit diesem Anspruch sieht die Erzählung die Einzigkeit Gottes, die Unversehrtheit des Kultes in Jerusalems und den jüdische way of life gefährdet. Das Zweite Makkabäerbuch greift genau die Elemente auf, die Antiochus zu seiner medialen Selbstinszenierung nutzt, bindet diese in eine hoch emotional, spannend und unterhaltsam erzählte Geschichte ein, um am Ende zu einem theologischen Ergebnis zu gelangen. Das Ergebnis lautet: Nicht der König Antiochus ist ein Theos Epiphanes, sondern der einzig wahre und epiphane König ist der Gott Israels. Dieses Ergebnis können die Leserinnen und Leser nun nicht nur intellektuell, sondern viel tiefer nachvollziehen, weil die Erzählung so konzipiert ist, dass sie ihr mit großer emotionaler Anteilnahme folgen können.

Am Beispiel des Epiphanie-Motivs kann man somit zeigen, dass sich das Zweite Makkabäerbuch mit der hellenistischen Gesellschaft und Kultur kritisch auseinandersetzt und das Porträt des eigenen Gottes in differenzierter Weise im Dialog mit der dominierenden Kultur profiliert. ${ }^{85}$

\section{Bibliographie}

BARAG, D., "The Mint of Antiochus IV. in Jerusalem. Numismatic Evidence on the Prelude to the Maccabean Revolt", in: INJ 14 (2000/2001) 59-77.

BARTLETT, J.R., The first and second Book of Maccabees, Cambridge: Harvard University Press, 1973.

BÉVENOT, H., Die beiden Makkabäerbücher, Bonn: Hanstein, 1931.

85 Zur Verhältnisbestimmung von Judentum und hellenistischer Welt mit Blick auf das zweite Makkabäerbuch: SCHWARTZ, 2 Maccabees, 52.66: Juden, die in der hellenistischen Welt leben, sehen "no contradiction between their being Jews and being Hellenized [...]. We term this synthesis 'Jewish-Hellenistic', but for our author it was simply 'Judaism', which was a legitimate and respected way to be Greek". 
BEVAN, E.R., „A Note on Antiochus Epiphanes “, in: JHS 20 (1900) 26-30.

BICKERMAN, E.J., “Héliodor au temple de Jérusalem”, in: Armuaire de l'Institut de Philologie et d'Historire Orientales et Slaves Gehrke (1939-1944) 7-40.

BICKERMAN, E.J., Studies in Jewish and Christian History, 2, Leiden u.a.: Brill, 1980, 15991.

BRINGMANN, K., Geschichte der Juden im Altertum. Vom babylonischen Exil bis zu arabischen Eroberung, Stuttgart: Klett-Cotta, 2005.

BUNGE, J.G., “Die sogenannte Religionsverfolgung Antiochus IV. Epiphanes und die griechischen Städte", in: JSJ 10 (1979) 155-65.

BunGE, J.G., "'Theos Epiphanes'. Zu den ersten fünf Regierungsjahren Antiochus IV. Epiphanes von Syrien", in: Historia 23 (1974) 57-85.

DORAN, R.M., Temple Propaganda: The Purpose and Character of 2 Macc (CBQ.MS 12), Washington, D.C.: Catholic Biblical Association of America, 1981.

EGO, B., God's Justice. The "Measure for Measure" Principle in 2 Maccabees, in: XERAVITS, G.G./ ZSENGELLÉR, J. (Hg.), The Books of Maccabees. History, Theology, Ideology (JSOTSup 118), Leiden: Brill, 2007, 141-54.

EHLING, K., Untersuchung zur Geschichte der späten Seleukiden (164-63 v.Chr.). Vom Tod des Antiochos IV. bis zur Einrichtung der Provinz Syria unter Pompeius (Historia Einzelschriften 196), Stuttgart: Steiner, 2008.

ENGEL, H., Die Bücher der Makkabäer, in: ZENGER, E. (Hg.) u.a, Einleitung in das Alte Testament (Kohlhammer Studienbücher Theologie 1,1), Stuttgart: Kohlhammer, 72008.

FISCHER, T., Heliodor im Tempel von Jerusalem. Ein hellenistischer Aspekt der „frommen Legende“, in: SIEGFRIED, H. (Hg.), Prophetie und geschichtliche Wirklichkeit im alten Israel, Stuttgart: Kohlhammer, 1991, 121-38.

FLEISCHER, R., Studien zur seleukidischen Kunst 1: Herrscherbildnisse, Mainz: von Zabern, 1991.

FRENSCHKOWSKI, M., Offenbarung und Epiphanie 2: Die verborgene Epiphanie in Spätantike und frühem Christentum (WUNT 80), Tübingen: Mohr, 1997.

FUNCK, B., “Herrscherkult der Seleukiden - Religionen einer Elite oder Reichsideologie? Einige Bemerkungen zur Fragestellung", in: Klio 73 (1991) 402-07.

GEHRKE, H.-J., "Der siegreiche König. Überlegungen zur hellenistischen Monarchie", in: Archiv für Kulturgeschichte 64 (1982) 247-77.

HABICHT, C., 2. Makkabäerbuch (JSHRZ 1,3), Gütersloh: Mohn, 1976.

HerR, B., “Der Standpunkt des Epitomators. Perspektivenwechsel in der Forschung am Zweiten Makkabäerbuch", in: Bib 90 (2009) 1-31.

HoOver, O.D., Coins of the Seleucid Empire from the Collection of Arthur Houghton, Part II, New York: The American Numismatic Association, 2007.

Houghton, A., Coins of the Seleucid Empire from the Collection of Arthur Houghton, New York: The American Numismatic Association, 1983. 
Houghton, A./ LORBER, C., Seleucid Coins. A Comprehensive Catalogue, 21: Seleucus IV through Antiochus XIII, New York: The American Numismatic Association, 2008.

HowgEGO, C., Geld in der antiken Welt, Darmstadt: Wissenschaftliche Buchgesellschaft, 2000.

HÜBNER, U., Tradition und Innovation. Die Münzprägung der Hasmonäer des 2. und 1. Jahrhunderts v. Chr. als Massenmedium, in: Frevel, Ch. (Hg.), Medien im antiken Palästina. Materielle Kommunikation und Medialität als Thema der Palästinaarchäologie, Tübingen: Mohr Siebeck, 2005, 171-87.

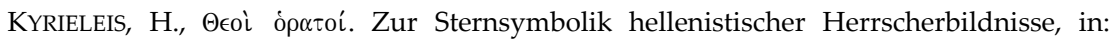
BRAUN, K.U./ FURTWÄNGLER, A. (Hg.), Studien zur klassischen Archäologie, FS D. Hiller (Saarbrücker Studien zur Archäologie und Alten Geschichte 1), Saarbrücken: Saarbrücker Druck und Verlag, 1986, 55-72.

LE RIDER, G., Antioche de Syrie sous les Séleucides. Corpus des Monnais d'or et d'argent I, Paris: Institut de France, 1999.

MitTAG, P.F., Antiochos IV. Epiphanes. Eine politische Biographie (Klio.B Neue Folge 11), Berlin: Akademie Verlag, 2006.

MørKholm, O., Studies in the Coinage of Antiochus IV of Syria, Kopenhagen: Munksgaard, 1963.

NICKLAS, T., “Der Historiker als Erzähler. Zur Zeichnung des Seleukidenkönigs Antiochus in 2Makk. IX", in: VT 52 (2002) 80-92.

NILSSON, M.P., Geschichte der griechischen Religion, 2: Die hellenistische und römische Zeit, München: C.H. Beck, ${ }^{2} 1961$.

NunN, A., Bilder als Massenmedien in Phönizien, Syrien und Jordanien vom 6. bis zum 4. Jahrhundert v.Chr., in: UeHLINGER, Ch. (Hg.), Images as Media. Sources for the Cultural History of the Near East and the Eastern Mediterranean (1 ${ }^{\text {st }}$ Millenium BCE) (OBO 175), Fribourg/ Göttingen: University Press, 2000, 359-74.

RAJAK, T., Jewish Perspectives on Hellenistic Rulers (Hellenistic Culture and Society 50), Berkeley u.a.: UC Press, 2007.

SCHMITZ, B., Auferstehung und Epiphanie. Jenseits- und Körperkonzepte im Zweiten Makkabäerbuch, in: NICKLAS, Th. (Hg.) u.a., The Human Body in Death and Resurrection (Deuterocanonical and Cognate Literature Yearbook 2009), Berlin/ New York: de Gruyter, 2009, 105-42.

SCHMITZ, B., “Geschaffen aus dem Nichts? Die Funktion der Rede von der Schöpfung im Zweiten Makkabäerbuch", in: NICKLAS, T./ ZAMFIR, K. (Hg.), Theology of Creation in Early Judaism and Ancient Christianity, FS H. Klein (Deuterocanonical and Cognate Literature Studies 6), Berlin/ New York: de Gruyter, 2010, 61-79.

SCHMITZ, B., Prophetie und Königtum. Eine narratologisch-historische Methodologie entwickelt an den Königsbüchern (FAT 60), Tübingen: Mohr Siebeck, 2008.

SCHWARTZ, D.R., Why Did Antiochus Have to Fall (II Maccabees 9:7), in: LiDONNICI, L./ LIEBER, A. (Hg.), Heavenly Tablets. Interpretation, Identity and Tradition in Ancient Judaism, Leiden u.a.: Brill, 2007, 257-65. 
SCHWARTZ, D.R., 2 Maccabees (Commentaries on Early Jewish Literature), Berlin/ New York: de Gruyter, 2008.

SCHWARTZ, D.R., From the Maccabees to Masada: On Diasporan Historiography of the Second Temple Period, in: OpPEnHEIMER, A. (Hg.), Jüdische Geschichte in hellenistisch-römischer Zeit. Wege der Forschung: Vom alten zum neuen Schürer, München: Oldenbourg, 1999, 29-40.

SEIDL, U., Babylonische und assyrische Kultbilder in den Massenmedien des 1. Jahrtausends v Chr., in: UeHLINGER, Ch. (Hg.), Images as Media. Sources for the Cultural History of the Near East and the Eastern Mediterranean (1 ${ }^{\text {st }}$ Millenium BCE) (OBO 175), Fribourg/ Göttingen: University Press, 2000, 89-114.

Van Henten, J.W., The Maccabean Martyrs as Saviours of the Jewish People. A Study of 2 and 4 Maccabees, Leiden u.a.: Brill, 1997.

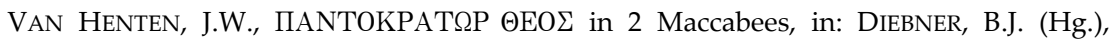
Yhwh - Kyrios - Antitheism or the Power of the Word, FS R. Zuurmond, Amsterdam/ Heidelberg: Selbstverlag des DBAT 1996, 117-26.

VAN HENTEN, J.W., Das Jüdische Selbstverständnis in den Ältesten Martyrien, in: VAN HENTEN, J.W. (Hg.), Die Entstehung der Jüdischen Martyrologie (StPB), Leiden u.a.: Brill, 1998, 127-61.

WEISER, B., “Das erste Massenmedium", in: Welt und Umwelt der Bibel 47 (2008) 34-37.

WeITZMAN, S., "Plotting Antiochus's Persecution", in: JBL 123 (2004) 219-34.

ZANKER, P., The Power of Images in the Age of Augustus, Ann Arbor: University of Michigan Press, 1988.

ZimmermanN, C., Die Namen des Vaters. Studien zu ausgewählten neutestamentlichen Gottesbezeichnungen vor ihrem frühjüdischen und paganen Sprachhorizont (Ancient Judaism and Early Christianity 69), Leiden u.a.: Brill, 2007. 